\title{
El pensamiento geográfico como acción y como resultado. Las relaciones entre la producción de un modo específico de pensar y la generación de conocimiento científico
}

\author{
Geographical Thinking as Action and as a Result. the \\ Relationships Between the Production of a Specific Way \\ of Thinking and the Generation of Scientific Knowledge
}

\author{
Abraham Paulsen Bilbao' (1)
}

\begin{abstract}
RESUMEN
Se presenta al pensamiento geográfico como acción y como producto o resultado. Sostenemos que toda ciencia genera en quienes las profesan un tipo específico de pensamientos, comprendidos como procesos mentales que influyen desde una perspectiva específica en la percepción u observación, análisis, explicación de problemas del entorno. Si se trata de acciones, surge la necesidad de reflexionar acerca de las formas como lo espacial influye en lo mental, si ambas dimensiones son estancos separados o se encuentran inter - compenetrados y si tales pensamientos implican la producción de representaciones, especialmente en lo concerniente a la aprehensión de las cualidades de los objetos. En tanto producto o resultado, el pensamiento geográfico equivale a un cúmulo de conocimientos que denominamos pensamiento geográfico, desde el cual se constituyen teorizaciones, herramientas, estrategias y modelos que aportan al ser humano competencias para relacionarse con el mundo. Aplicamos en la determinación de qué significa pensar geográficamente algunos insumos provenientes de la Filosofía de la Mente, de la Teoría Representacional y de la Neurociencia. Además, muy sucintamente, reflexionamos acerca de las implicancias de esta concepción de pensamiento geográfico en la formación de los futuros geógrafos y geógrafas.
\end{abstract}

Palabras claves: Pensamiento Geográfico - Mente - Representaciones - Filosofía de la mente - Neurociencias

\section{ABSTRACT}

It is presented to geographical thinking as an action and as a product or result. We maintain that all science generates in those who profess it a specific type of thoughts, understood as mental processes that influence from a specific perspective in the perception or observation, analysis, explanation of problems of the environment. If we are dealing with actions, the need arises to reflect on the ways in which the spatial influences the mental, if both dimensions are separate or interpenetrated and if such thoughts imply the production of representations, especially with regard to the apprehension of the qualities of 


\begin{abstract}
objects. As a product or result, geographic thinking is equivalent to an accumulation of knowledge that we call geographic thinking, from which theorizations, tools, strategies and models are constituted that provide the human being with competencies to relate to the world. We apply in the determination of what it means to think geographically some inputs coming from the Philosophy of Mind, from the Representational Theory and from Neuroscience. In addition, we briefly reflect on the implications of this conception of geographic thinking in the formation of future geographers.
\end{abstract}

Keywords: Think Geographically - Mind- Representations - Philosophy of the mind - Neurosciences

Todo ser humano cuenta con capacidades basadas en estructuras mentales mediante las cuales construye experiencias, interviene e interpreta el mundo. El cerebro es el soporte o estructura básica material responsable de la construcción de procesos abstractos que aglutinamos bajo la concepción de lo mental, produciendo representaciones y conocimientos, para lo cual evolucionó coherentemente con el mundo físico (Chopra, 2013; Penrose, 1994).

Los procesos biofísicos que sostienen la vida y lo mental son ámbitos que fenomenológicamente forman parte del mundo físico, objeto de estudio de la geografía, que aborda problemáticas asociadas a las vinculaciones entre las diversas formas de vida con las transformaciones socioespaciales. Considerando lo mental, nos proponemos reflexionar acerca de las propiedades fenomenológicas básicas que forman parte de lo que definiremos como pensamiento geográfico, que presenta matices como acción y como producto o resultado.

Sostenemos que toda ciencia construye un tipo específico de pensamiento coherente con los problemas centrales que aborda y que se evidencia en acciones, resultados y representaciones; desde la geografía nos interesa estudiar los aspectos materiales e inmateriales que influyen en la producción de un tipo específico de saber o conocimiento, entendido como un acervo de teorías, conceptos, métodos, textos, discursos e investigaciones. Nos proponemos responder a las siguientes interrogantes, ¿existe un pensamiento propiamente "geográfico" ?, y de existir, ¿cuáles serían sus características?, ¿qué implicancias podrían tener este tipo de pensamientos en el proceso de transmisión intergeneracional de lo geográfico y en la formación de futuros científicos?

Para responder estas interrogantes y otras asociadas, decidimos primero separar al pensar como acción y como resultado; en tanto es acción interesan sus relaciones con la mente y con el cerebro y como resultado, nos referiremos al desarrollo de conocimiento y de habilidades geográficas. Al respecto, como es de suponer, se han instalado diversos planteamientos que fluctúan desde la plena separabilidad hasta la más absoluta unicidad; independientemente de las visiones en conflicto, la percepción de que cuando pensamos hacemos algo independiente de las funciones cerebrales es una situación que permea a todas las teorías y modelos vigentes y también influye poderosamente la existencia de la sensación a la que tradicionalmente llamados autoconciencia (Jonas, 2000; Merlan \& Jonas, 1967; Rubenstein, 2009).

Los descubrimientos de la neurociencia contemporánea reactivaron las discusiones disciplinares e interdisciplinares referidas a las relaciones entre mente y cerebro, este último entendido como la unidad biológica que procesa e integra los estímulos internos y externos que recibe produciendo un tipo específico de cognición orientada a la producción de una reacción o respuesta (Searle, 1985; Thagard, 2019). Respecto a la mente, aludimos a la experiencia cognitiva resultante 
de la suma de procesos psíquicos cognitivos o afectivos, conscientes e inconscientes, que derivan de la actividad cerebral, responsable de la existencia de actividades mentales. La existencia de procesos psíquicos conscientes e inconscientes instala como punto de partida en la discusión acerca de la semejanza o diferencia entre actividades mentales y funciones o procesos cerebrales al problema de la consciencia, concebido como eje analítico de las relaciones mente - cerebro y mente -cuerpo (Pinker, 2012; Tirapu-Ustárroz et al., 2007).

Segundo, nos centraremos en el objeto, en el pensar geográficamente basándonos fundamentalmente en la teoría representacional, ya que todo pensamiento es una representación consciente o inconsciente, voluntaria o involuntaria que resulta de las relaciones ser humano - sociedad - medio.

El pensamiento geográfico produce representaciones referidas a hechos, fenómenos y elementos de la naturaleza que pertenecen al conocimiento geográfico, lo cual implica que el acto de pensar y el conocer desde la geografía al mundo existe una interdependencia donde un aspecto es necesario para que exista el otro y en los que las representaciones permiten analizar, discriminar, explicar procesos aplicando teorías científicas sobre la base de abstracciones, hipótesis y teorizaciones (Nickerson, R.S, Perkins, D.N, Smith, 1994; Perner, 1997). Entonces, pensar geográficamente es representar y representar-se bajo la forma de algo que mantiene una relación (de representación, reemplazo, en lugar de) con otra cosa.

El contenido de ese pensar es lo que representa algo como es, que lo reemplaza bajo la forma de otra sustancia, que emerge desde un ente biológico - químico - físico anidado en el cerebro. Dada la necesidad del medio representacional para que el pensamiento aparezca, la representación mental o el pensar es materialmente una intención de comunicación por parte de un sujeto (Grice, 1957) basándose en un sistema (representacional) cuya función es indicar de qué manera las cosas están en relación con otros objetos, condiciones y su respectiva magnitud (Dretske, 1988).

En la tercera sección vinculamos al pensamiento geográfico con la producción de representaciones, lo cual vincularemos con una cuarta sección donde nos centraremos en la experiencia del aprendizaje en la formación de futuros geógrafos. Por último, incorporamos algunas conclusiones, proponiendo, de paso, puntos que podrían ser trabajados por investigaciones y trabajos posteriores.

\section{El pensamiento geográfico como acción; estructuras cognitivas implicadas en un proceso complejo, dinámico y transformador}

Las neurociencias han profundizado el análisis de los elementos estructurales que influyen en el desarrollo del pensamiento geográfico, centrándose en las posibles influencias que genera el entorno en los procesos de percepción y en la actividad cerebral (Mainzer, 1994). Además, esta disciplina aportó a la determinación de una especie de sistema de navegación neuronal cuya operación depende de dos tipos de células específicas, las células del lugar y las células de red. 
Las primeras codifican la posición y las segundas se activan cuando pasamos por algún sitio, mapeando para hacer posible el reconocimiento del lugar y seleccionar las mejores posibilidades de desplazamiento. Estas neuronas se distribuyen en distintas regiones cerebrales, principalmente en el Hipocampo y en la Corteza Entorrinal del Lóbulo Temporal Medio (Aguilar, 2015).

Otro aspecto descrito por los neurocientíficos referido a cuestiones estructurales asociadas al pensamiento geográfico es el hecho de que la incorporación de contenidos o temáticas geográficas en un proceso de aprendizaje genera cambios cerebrales. Un estudio publicado a inicios del presente siglo mostró aumento en el tamaño del Hipocampo posterior en adultos que aprobaban, tras tres o cuatro años de preparación, un examen que los calificaba para la labor de taxistas en la ciudad de Londres, que demandaba la memorización de la localización de aproximadamente 25000 calles o lugares; se evidenciaba que el aprendizaje cambiaba estructuralmente el cerebro y posibilitaba el desarrollo de la memoria espacial (Maguire et al., 2000). De paso, los resultados de esta investigación pusieron en entredicho la inutilidad de los aprendizajes memorísticos y la rigidez de las estructuras comprometidas en el desarrollo de la memoria.

La flexibilidad estructural del cerebro y especificidades neuronales y regionales como las descritas son también respaldadas por otros trabajos provenientes desde la psicología cognitiva (Claxton, 1996, 2002, 2007; Medina C., 2014; Stern, 2015) y desde la teoría de los engramas (Palm, 1981; Rashid, A.J.; Yan, C.; Mercaldo, V.; Hsiang, H.; Park, S.; Cole, C.J.; De Cristofaro, A.; Yu, J.; Ramakrishnan, C.; Lee, SY., Deisseroth, K.; Frankland, P.W.; Josselyn et al., 2016; Shaw, 1986).

La evolución actual de las neurociencias define a fenómenos mentales conscientes tales como pensamiento y consciencia como funciones emergentes de la actividad global del cerebro y muy especialmente del neo córtex, reconocidos como niveles superiores del cerebro (Searle, 1985; Thagard, 2019). Por ende, según Searle, lo que llamamos mental es un fenómeno esencialmente biológico que depende de una función sistemática y holística del sistema nervioso, sin que por ello se encuentre aparte en otro lugar o espacio (Searle, 1996). La teoría neurocientífica actual sustenta esta tesis en el sentido de que los fenómenos mentales corresponden a la expresión biológica de los procesos cerebrales (Medina C., 2014), lo cual pone en riesgo el rol de la conciencia, cuya existencia ya no es necesaria si concebimos que el cerebro en tanto organismo biológico funciona produciendo respuestas integrando estímulos (Chalmers, 2002) y por otra parte, este enfoque dificulta la explicación acerca de la naturaleza y características tanto de los estados mentales como de las percepciones y también las razones por las cuales los estados presentan importantes diferencias en su intencionalidad y carácter subjetivo.

De acuerdo a lo anterior, la conciencia humana se caracteriza por la posesión de doce atributos, siendo el primero el referido a que se manifiesta en modalidades finitas corporales y de flujos de pensamiento, unidad o sincronía horizontal y vertical, intencionalidad, sentimiento subjetivo de nuestros estados conscientes, vinculación entre intencionalidad y conciencia, estructura gestáltica, familiaridad entre estados consientes que posibilita la organización y orden de las experiencias conscientes, desbordamiento, presencia de un centro y de una periferia, existencia de condiciones límite, estados de ánimo y de la dimensión places y displacer. Todos estos rasgos perfilan a la conciencia como un fenómeno cerebral a macro nivel emergente de lo físico cuya realidad y apariencia depende tanto de la subjetividad como de las percepciones y del flujo de 
pensamiento que tenga el sujeto consciente (Bennett, Maxwell; Dennett, Daniel; Hacker, Peter; Searle, John, Robinson, 2003; Searle, 1994, 1996; Singer \& Lamm, 2009).

Como es el caso de otras teorías, este tipo de planteamientos han sido debatidos, criticados y considerados insuficientes, materialistas y parciales desde diversas perspectivas y autores (G. Edelman, 2006; G. M. Edelman, 2006; Mauro \& Edelman, 2002; Varela, 1996, 1999), pero existe consenso en que pese a las objeciones descritas, significaron un avance importante en la comprensión y definición del proceso de pensamiento en dependencia de un tipo específico de filosofía de la mente que se desentiende de las nociones tradicionales de representación computacional del pensar y de modelos conductistas, comprendiendo al cerebro en interacción no lineal con el cuerpo y el mundo.

Desde otra mirada, la filosofía de la mente, donde destacaremos las obras de John Searle (Searle, 1985, 2013), Gilbert Ryle (Ryle, 2005, 2014), Daniel Dennett (D. Dennett, 2009; D. C. Dennett, 2012) incluye a los aprendizajes geográficos y/o espaciales en el conjunto de los conceptos disposicionales que forman parte de enunciados semi - hipotéticos. En este contexto, los conceptos asociados a conductas espaciales se asocian con todas aquellas palabras que utilizamos cuando describimos y explicamos la conducta humana. Se trata generalmente de disposiciones o actitudes no episódicas que se manifiestan repetitivamente y no solo en un determinado momento, siendo por lo tanto aspectos más o menos permanentes de la conducta que afloran en un individuo ante diversas situaciones y que se oponen a las conductas extraordinarias en actos discontinuos que son descritas mediante palabras episódicas (Ryle, 2005).

Avanzando un poco más en las filosofías de la mente y reconociendo el carácter no lineal de los procesos de formulación de pensamientos, sostenemos que pensar geográficamente implica el establecimiento de relaciones bidireccionales o recíprocas entre eventos neuronales y actividad consciente en coherencia con el atributo de emergencia inherente a los sistemas complejos y que más que tratarse de la consecuencia de un proceso únicamente cerebro - neuronales, pensar geográficamente vincula al cerebro con el cuerpo y con el mundo. Por otra parte, la Neurociencia Cognitiva ha demostrado que pensamientos específicos devienen de la integración episódica de numerosas áreas cerebrales distribuidas en distintos sectores y que desarrollan permanentemente diversos niveles y formas de interacción sin despegarse del cuerpo y del mundo, ya que estos tres componentes son sistemas dinámicos muy estructurados acoplados entre sí de tal manera que es difícil separar lo "interno" de lo "externo" (Thompson \& Varela, 2001; Varela, 1996, 1999).

Thompson y Varela sustentaron la tesis de que las interacciones descritas posibilitaban la emergencia como causalidad ascendente de procesos globales del organismo con el medio que influían en los elementos constituyentes bajo la forma de causalidad descendente. A juicio de los autores, las influencias se expresan en tres ciclos o dimensiones en los animales superiores: regulación organísmica, acoplamiento sensorimotor entre organismo y medio, interacción intersubjetiva (Thompson \& Varela, 2001).

El ciclo de regulación organísmica incluye a todo el cuerpo y el cerebro interviene sobre la base del funcionamiento de procesos neuronales del sistema nervioso autónomo que vinculan los sensores y efectores corporales con los mecanismos homeodinámicos básicos de los organismos internos y las vísceras. Estas relaciones originan los estados emocionales y sentimientos afectivos 
típicos de los mamíferos definidos como conciencia central o primaria, que describe la sensación que todos hemos tenido de que estamos vivos y que tenemos en cada estado consciente correspondencia con un contexto afectivo ineludible que aporta al desarrollo de la identidad (Panksepp, 1998, 2007).

El ciclo de acoplamiento sensorimotor relaciona al sujeto con su entorno entregando información acerca del movimiento, que es función de lo que siente y que le faculta para comprender que es un agente situado. Este ciclo se despliega mediante el concurso de las vías sensorio motoras del cuerpo que se acoplan con el entorno. La interacción subjetiva se relaciona con el desarrollo de la cognición social, la que a su vez depende de las dimensiones anteriores y requiere del acoplamiento sensorio motor de neuronas que son fundamentales tanto para la cognición social como para el desarrollo de las emociones y presumiblemente, del lenguaje (Rizzolatti \& Arbib, 1998).

Ya que nos referimos al lenguaje, nos detendremos en una breve consideración. Definimos a la lingüística como el estudio concreto de las estructuras mentales que subyacen en el desarrollo del lenguaje entendido como una capacidad de segundo orden, lo descartamos como un insumo válido para analizar las estructuras mentales que intervienen en el pensamiento geográfico, cuestión que nos distingue de otras posturas que pretenden abordar desde el problema del lenguaje las estructuras mentales que lo producen y desde estas a todas las existentes en el cerebro. En lo que respecta al ámbito geográfico, tanto las habilidades como la cognición asociadas a lo espacial dependen de estructuras cuya comprensión requieren del complemento de más datos que los que el análisis lingüístico otorga.

Por lo anterior, afirmamos que pensar es identificar e integrar al lenguaje mediante una operación intelectual individual que puede ser voluntaria o no, dependiendo de la existencia de racionalidad o la acción de un estímulo que deriva en una elaboración mental. El lenguaje es al pensamiento tanto un modo de producción como de transformación y conservación (Bruner, 2003; Londoño, 2010).

\section{El pensamiento geográfico como resultado; la geografía como aprendizaje y acervo de memoria}

Cuando decimos que alguien es competente en determinada profesión u oficio, estamos evaluado positivamente sus logros y desempeños en tareas episódicas, diversas, para las cuales se formó y cuyos desempeños mejoraron con la práctica. Desde hace largo tiempo, la profesionalidad competente de las nuevas generaciones depende de la trasmisión cultural de habilidades, conocimientos y actitudes desde una generación a otra en un proceso de transferencia de conocimientos desde un cerebro más informado a otro más desinformado y que requiere ser capacitado en un determinado ámbito científico o técnico, derivando en teorías, procedimientos, técnicas, tecnologías, conceptos y significados, vale decir, pensamientos materializados destinados a explicar la realidad.

Gabriel Tarde, Walter Benjamin, Michel Foucault, entre otros, reflexionaron acerca del pensamiento como resultado o materializado, asociándolo a la producción de explicaciones acerca de la realidad, ligándolo con la facultad mimética. Mientras que Gabriel Tarde analizó las relaciones 
entre poder y mímesis, suscribiendo esta última al análisis de la realidad a partir de la comparación mediada por las dinámicas socioespaciales concurrentes (Barry \& Thrift, 2007; Latour, 2012; Tarde, 2013; Toscano, 2007), Walter Benjamin definió a la mímesis como facultad que permitía al ser humano la creación de sistemas de correspondencias o redes de equivalencias entre entidades que pueden o no ser similares desde una perspectiva sensorial; según Benjamin, la facultad mimética era un método transhistórico que a raíz de la evolución en las técnicas incrementaba sus posibilidades de aplicación y explicación (Benjamin, 2010).

Michel Foucault, al reflexionar acerca de los campos epistémicos y regímenes discursivos imperantes desde la época clásica, marcó las diferencias entre el formalismo científico anterior al siglo XVIII y el que se constituyó a partir de la Modernidad. Concibió a la producción científica de conocimiento, comprensión y explicación de la realidad previa a la llustración como el resultado de cuatro operaciones básicas de la mímesis: convenientia, aemulatio, analogía y simpatía, factores operantes en la construcción de la realidad mediante un complejo sistema de correspondencias, relaciones, sucesiones, reflejos y reciprocidades (Foucault, 1978, 1985, 2008).

En los tres autores señalados la mímesis aparece como una postura acerca del conocimiento mediada por las relaciones de poder, fundamento de una episteme que varió a partir del siglo XVIII y como aspecto central en la configuración de representaciones (Benjamin, 2010; Foucault, 1978, 2008; Melero Martínez \& Blanco Mayor, 1991; Tarde, 2013; Thrift, 2004).

En lo que concierne al pensamiento geográfico, desde la facultad mimética surge las concepciones de espacio geográfico como el producto de la agencia de los atributos de distancia y separación derivados de la imposibilidad de que dos cuerpos tengan una localización idéntica en un punto o lugar al mismo tiempo (Lussault, 2015). Por otra parte, siguiendo a Foucault, la facultad mimética, la interacción entre la antipatía y la simpatía como conflicto de poder decisivo en la singularidad o desaparición de la identidad, y la cuestión referida a las vinculaciones entre espacialidad, temporalidad y lenguaje, actúan en la producción de espacios lugarizados correspondientes con otras cronologías de pensamiento (Foucault, 1985; Porto-Gonçalves, 2009).

Desde el siglo XVIII la geografía comenzó a constituirse como un conocimiento discernible en el desarrollo de las ciencias y que evolucionó desde los paradigmas deterministas y posibilistas para alcanzar, según Edgar Morin, un carácter trans e interdisciplinario, atributos que son posibles por su condición de ciencia multidimensional y compleja (Morin, 1999). El estatus descrito distingue a lo geográfico como un tipo específico de reflexión no reduccionista que, gracias al auge de la ecología y otras ciencias sistémicas que abordan el estudio de la Tierra, recuperó en buena hora sus cualidades multidimensionales, complejas y totalizadoras, por lo que ha alcanzado un lugar privilegiado en el ámbito de las Ciencias de la Tierra.

La constitución de la geografía moderna durante las postrimerías del siglo XVIII y el largo siglo XIX, supuso la maduración del pensamiento geográfico sobre la base de la discusión teórica acerca de las relaciones ser humano - sociedad - medio y de la construcción de los conceptos estructurantes de la disciplina y que orientan al pensamiento geográfico hasta nuestros días. Este proceso se dio coetáneamente con el paso desde los métodos por los cuales las ciencias buscaban descubrir los secretos del Universo mediante la búsqueda de patrones, semejanzas, marcas o signos presentes tanto en el micro como en el macrocosmos, a la producción de taxonomías, 
categorías y clasificaciones, construcción de datos, que significó la separación definitiva entre magia y erudición.

El pensamiento geográfico se constituyó en un dispositivo que se movilizó, como la mayoría de los restantes conocimientos modernos, por la asociación entre saber - poder y verdad (Casey, 1987; Michel Foucault, 1985, 1992, 1993, 2000; Philo, 2011) y tuvo en la experimentación como insumo para la producción de conocimiento (Berman, 1987; Escobar, 2003; Latour, 1983). Entonces, pensar geográficamente, concebido como acción resultaba en la producción de un pensamiento geográfico que fue evolucionando hasta convertirse en un dominio trans e interdisciplinario que integraba tres dimensiones para producir un saber sabio que se aplica al estudio de las relaciones ser humano - sociedad - medio.

El desarrollo del pensamiento permitió la ejecución de acciones o movimientos que demandaban, por ejemplo, orientarse, interpretar un mapa, analizar un paisaje. Pero también se constituyó una estrategia cognitiva que, siendo permanente, una vez aprendida se incorporó a las diversas situaciones que constituyen la vida. Por eso, cuando decimos que alguien piensa geográficamente aludimos a un sujeto que tiene la capacidad de aplicar episódicamente conocimientos provenientes de la geografía y disciplinas afines como también que al haber recibido formación geográfica manifiesta un modo particular de enfocar las más diversas circunstancias de la vida, junto a hábitos y valores formados en parte, por ese tipo de conocimiento que produce una gama de acciones y reacciones diferentes pero predecibles según los dictados de la ciencia que las motiva.

\section{El Pensamiento Geográfico como un acto representacional}

Gabriel Tarde, Walter Benjamin, Arthur Schopenhauer, Michel Foucault, Henri Lefebvre, entre otros, abordaron el problema de las representaciones revisando las formas como tradicionalmente se le asoció al concepto de mímesis. En la Grecia Clásica Platón relacionó a la mímesis como un tipo específico de semejanza representacional; posteriormente, Aristóteles determinó que la mímesis era uno de los conceptos centrales de la filosofía y estética occidental, profundizó la asociación platónica con el concepto de representación o de representación mimética (Chomsky, 1983; Foucault, 1978; Jackson, 2004; Moscovici, 1984).

Desde mediados del siglo pasado, en el contexto de las ciencias humanas y sociales surgieron nuevos planteamientos en ámbitos tales como arte, cultura, cognición, sociedad, que postularon que el lenguaje representaba al mundo y relacionaron al discurso científico o al trabajo artístico con las concepciones de realidad (Araya, 2002; Moñivas Lázaro, 1994; Moscovici, 1984). Se impuso la teoría acerca de que la representación o imagen mental correspondía a uno de los accidentes que puede poseer una realidad o un objeto, que manifiesta dos tipos de atributos o cualidades, las llamadas primarias (por ejemplo, forma, extensión y el movimiento) y las secundarias (lo que perciben subjetivamente nuestros sentidos y que se comunican bajo la lógica de conceptos generalistas tales como apariencia, color, aroma, sabor y otras afines). 
Toda representación se asemejaría a un ensamble entre ambas formas de cualidades, de modo que se hace necesario resolver si estas, (las cualidades), están en los objetos o son únicamente construcciones mentales. Esta situación era especialmente importante en el análisis de las cualidades secundarias, por cuanto estas tienen un carácter esencialmente subjetivo. Surgieron dos posibilidades de respuesta, la primera esgrimida por los asocianistas, quienes postulaban que solo existían cualidades en los objetos, y quienes desde la psicología del Gestalt eran partidarios de que las cualidades correspondían a construcciones mentales, vale decir, producciones subjetivas (Kanai \& Tsuchiya, 2012; Nida-Rümelin, 2009; Shoemaker, 1991).

Walter Benjamin la definió como el aura del objeto representado, la trama singular de espacio y tiempo producida por el poder de la distancia o por la imposibilidad de que lo representado esté materialmente cuando es lenguajeado (Benjamin, 2007). Posteriormente Foucault analizó las representaciones en sus relaciones con la evolución de las ciencias humanas y sociales desde el siglo XVIII en adelante, definiendo, de paso, a las representaciones literarias y pictóricas en función de los aportes de las teorías miméticas (Foucault, 1985). Con una vinculación mayor con el pensamiento de Benjamin que con los aportes de Foucault, Didi-Huberman añadió al aura el carácter de espaciamiento obrado y hasta labrado; releva en la definición de Benjamin la condición de trama por cuanto es un acontecimiento único y extraño, a modo de trama de una obra, que nos atrapa atrayendo nuestra atención, lo que le hace también tejido. El aura entonces es un espaciamiento obrado y originario del mirante y del mirado, del mirante por el mirado (Didi-Huberman, 1997).

Una imagen representa una realidad de la que es parte, la cual es la obra. Siempre la imagen representacional está en "lugar de" y se origina "desde" o "de parte de" y es en si misma un pensar aislado o como el cuesco de una palabra usada para convocar, hacer presente una realidad que no está en el minuto en el que estamos comunicando procesos a ella inherentes. Por eso, representar también alude a "aparecer" mediante un medio físico apto para que algo que nos estaba velado se haga inmanente. El aparecer precisa de un aparato que haga señalable la presencia o representación, por ejemplo, el lenguaje, la palabra, el signo. Lo señalable, a su vez, produce un acontecimiento que renueva el valor y significancia del símbolo (Derrida, 1989; Perner, 1997).

Pensar geográficamente es un acto representacional cuyo resultado es un conjunto de nociones que evolucionan a conceptos, los que se enhebran en estructuras cognitivo - discursivas a las cuales denominamos teorías. Por otra parte, las representaciones son modelos de los cuales el lenguaje pasa a ser el medio representacional por el cual se proyectan los entes que son los contenidos de la representación. La representación mimética depende de la capacidad de captación de la mayor cantidad posible de atributos de la realidad por parte del producto representacional; tal percepción de un mundo fenomenológicamente dinámico cambia en coherencia con las variaciones en la posesión o en la perspectiva del sujeto. Se trata entonces de un proceso fundamental para la producción del cúmulo representacional desde el cual se construye el pensamiento geográfico, entendido como la suma de experiencias fenoménicas.

Las representaciones cartográficas y otras imágenes que aportan a la producción de conocimiento geográfico corresponden a este mismo tipo de experiencias, generando un tipo específico de pensamiento que reemplaza, a diversas escalas, al mundo natural y cultural que pretende representar. Los sistemas de representación geográfica son entonces modalidades de resignifi- 
cación y representación de la realidad que nutren al pensamiento geográfico sobre la base de convenciones técnicas y culturales que activan diversas formas de estados mentales.

Apelando a los estados mentales generados en las representaciones erróneas y a la diferencia entre saber, pensar y recordar, la psicología cognitiva ha buscado definir satisfactoriamente lo que es una representación o lo que hace que algo lo sea. En principio, el concepto alude a la obra filosófica de David Hume, John Locke, Henri Bergson, Gottlob Frege, Ludwig Wittgenstein, Jacques Derrida, entre otros, o a cosas tan distintas como imágenes externas, modelos, enunciados y estados mentales. Toda representación no es un objeto en sí mismo, sino que, en su capacidad representacional evocan siempre otra cosa. Perner determinó tres componentes de una representación: medio representacional, contenido representacional y relación de representación, atribuyendo al pensamiento el carácter de contenido o relación y al lenguaje el rol de medio (Perner, 1997).

Toda representación mental o pensamiento intencionado se da en un contexto del cual deriva su fuerza; es el contexto el que permite que aquello de lo cual se habla o del que de algún modo se hace referencia se haga visible en un lugar idóneo para que un proceso de remedo o imitación de la realidad "tenga lugar", o sea oportuno, pertinente y procedente. De lo anterior se deduce el atributo principal del pensar geográficamente que es posibilitar la sustitución mimética de la realidad, reconstruyendo (o reproduciendo) lo que no se encuentra presente, (en presencia), en el espacio tiempo en el cual establecemos algún tipo de relación cognitiva - comunicativa mediante el uso de un aparato o instrumento físico, conceptual, lingüístico, o de otro tipo desde el cual abordamos la tarea de reconstruir. Son diversas las ciencias y disciplinas que construyen aparatos conceptuales cuyo objetivo es precisamente el realizar procesos de sustitución mimética de la realidad, la geografía es una. Dentro de su campo y su método aparecen diversas formas como esta disciplina lleva a cabo tal desafío, y en todas los procesos son primero, dispositivos psicológico - cognitivos ya que es un individuo el que aprehende y traduce desde la representación aquello que se intentó representar.

La comunicación de una representación mental o pensamiento debe considerar que en toda relación de representación pueden generarse asimetrías, singularidades, representaciones erróneas y no existencias. Según Perner, las asimetrías se explican en la unidireccionalidad que define a las relaciones entre lo representado y su correspondiente representación; la singularidad se explica en que generalmente para tener sentido una representación se relaciona con un único objeto real; las representaciones erróneas se deben a nuestras incapacidades para representar correctamente todos los elementos de la realidad; se originan por un error perceptivo o porque un estado interno representa el sentido de algo de una manera que no es la correspondiente. Por eso, toda representación errónea surge cuando el sistema representacional no funciona correctamente. Por último, la categoría de no existencia reconoce que no todo lo que es real y que así y todo logramos construir imágenes fiables que podemos comunicar.

El pensar geográficamente se sustenta en diversos tipos de representaciones. Cuando dicho pensar es producido por entes con los cuales el sujeto tiene una relación causal directa que influye en que posee un alto nivel de conocimiento y capacidad lingüística para definir y representar lo que dicha cosa es, se produce entonces una representación primaria dada su imbricación con el mundo representado. Cuando representamos las cosas como creemos que debieran ser estamos separándonos voluntariamente de la realidad para configurar representaciones secundarias, cuya 
producción es exclusiva de los animales superiores y posibilitan considerar el pasado, el futuro posible, para el razonamiento hipotético, y para evaluar incluso aquello que no existe. Este tipo de representaciones son parasitarias de las primarias (Hall, 1980).

Entre las representaciones primarias y secundarias acontece una relación casi simbiótica, ya que sin el nexo causal con la realidad representada en el funcionamiento primario, los estados internos carecerían de función representacional ya que es lo primario quien otorga sentido a los estados internos que se desdoblan con el fin de producir representaciones en una función secundaria y metarrepresentaciones o representaciones de las representaciones, entendidas como marcas que dirigen el uso interno y que, en consecuencia, permiten diferenciar lo real de lo hipotético.

La geografía científica se constituye a partir de representaciones secundarios y se vale de modelos para explicar el comportamiento de los objetos y sujetos naturales y culturales, que son permanentemente reemplazados por pensamientos intencionados que dan cuenta de su existencia y que se construyen a partir de cuatro elementos: recursividad, subjetividad, objetividad y espacialidad. Lo recursivo se asocia a la producción de representaciones secundarias, las cuales, como ya señalamos, dependen de la existencia previa de otras más ligadas con la realidad; Humberto Maturana planteó que hay una recursión siempre que el observador o usuario puede afirmar que la replicación de una operación ocurre como consecuencia de su aplicación previa, a diferencia de las repeticiones, que dependen de que una operación dada se realice de nuevo independientemente de las consecuencias de su previa realización (Maturana, 1988, 2002).

La subjetividad afirma la gran dependencia que tienen las formas de pensamiento geográfico con la historia de vida individual y también colectiva donde tanto el conocedor como el medio experimentan cambios estructurales congruentes hasta que el sistema viviente muere. La objetividad alude a que toda representación primaria surge desde la conexión entre lo mental y lo real. Por último, la espacialidad u oportunidad se relaciona con el hecho de que es la sociedad en un espacio - tiempo determinado la que asume como verdadera o falsa una sentencia o un producto de su propio conocimiento, así como también define si una pregunta es o no oportuna. Por lo tanto, más que respuestas verdaderas o falsas, son las preguntas que se aceptan como verdaderas y las que se descartan por ser falsas las que estructuran la historia del conocimiento social situado. Los cuatro aspectos consignados otorgan al pensar la capacidad de transformar la realidad y a su vez se construyen a partir de la influencia del medio que también transforma al individuo que conoce el cual reorganiza el conjunto de su organización sensorial y modifica la estructura de su personalidad, por cuanto conocer equivale a adquirir o integrar información con el fin de describir o definir lo conocido señalando sus características o notas esenciales, relaciones con otros objetos y su función en el dominio de las experiencias.

Desde la perspectiva espacial señalamos que todo pensamiento está situado o lugarizado. El contexto o core tiene un rol fundamental en la emergencia del pensamiento y en la construcción de sus dimensiones representacionales. Ello explica la existencia de cosmovisiones y la diferencia entre culturas (Bale, 2006; Tuan, 2007).

Lo anterior coincide con el hecho que desde la psicología surgió el principio de que "son las representaciones del mundo, y no el mundo en sí, las que determinan la conducta"(Pozo, 1999, p. 
122), aludiendo al hecho de que todo pensamiento surge desde un estado mental que aborda lo real simplificando su condición múltiple informando la situación real del objeto representado o desplegándose en un conjunto de situaciones hipotéticas elaboradas a partir de una determinada realidad.

En este segundo ámbito, las representaciones responden a estados de deseos, asociándose a estados mentales conativos, tales como deseos necesidades o metas. Estos estados tienen especial incidencia en las conductas a seguir por un individuo; de hecho, los estados mentales conativos tienen un rol relevante en el gobierno de las acciones que permitan lograr los fines deseados. Sostenemos entonces que personas y sociedades actúan de acuerdo a la representación que construyen de la realidad y que toda representación se explica, en parte, por la sociedad en la cual se construyó, sin que ello implique que exista "un único sistema representacional social", aún cuando puedan darse aproximaciones entre individuos que comparten espacios, historia y conocimientos, que son una forma de creación social por excelencia.

El proceso de sustitución de la realidad desde el pensar cuando se transfiere al lenguaje, situación por la cual el pensar geográficamente pasa a ser un producto, constituye un referente, esto es el conjunto de significados que diversas expresiones lingüísticas comparten, al cual aporta un sentido, entendido como el significado que lo especifica y diferencia. Será entonces el referente de una representación el qué ella representa y el sentido la cosa representada en tanto es de una determinada manera. El referente será también un objeto de representación, una denotación o un tema perteneciente a lo que denominamos o entendemos como lo geográfico y el sentido será el significado o el comentario de lo previamente encasillado. Por ello, para que exista un pensar y una decodificación de dicho acto, se hace necesaria la existencia de una comunidad lingüística o científica que le atribuya un significado y una interpretación al concepto o la expresión que alguien comunica a otro.

Dijimos anteriormente que el estilo de pensamiento establece las condiciones previas a cualquier cognición y determina que se puede considerar como una pregunta razonable y una respuesta verdadera o falsa; también facilita el contexto y traza los límites para cualquier juicio sobre la realidad objetiva. Postulamos que la característica esencial de las representaciones colectivas es que se hallan ocultas a los integrantes del colectivo de pensamiento y operan como un sistema conceptual que limita y controla la cognición individual de manera tan estricta que limita de manera importante, en algunos casos, las modalidades de comunicación transcultural. Dicho de otro modo, toda representación es también un proceso de comprensión social que surge desde una epistemología sostenida por una comunidad de intelectuales y que origina patrones para hacer de esa comprensión un instrumento de decodificación de la realidad.

\section{Las implicancias del pensamiento geográfico en la experiencia de enseñar geografía en el contexto de la formación de geógrafos}

Actualmente los procesos de formación universitaria buscan desarrollar competencias en los estudiantes, tanto generales como específicas, instrumentales, académicas disciplinares, per- 
sonales, transversales, entre las principales (Mérenne-Schoumaker, 1994, 2003). Tal objetivo demanda la integración de contenidos, procedimientos o habilidades, actitudes susceptibles de ser aplicadas en un ejercicio profesional específico, integrándolas previamente.

Hemos intentado mostrar que determinados atributos, como podría ser el pensar geográficamente, caracteriza a un individuo que tiene competencias para aplicar una modalidad especifica de pensamiento en situaciones problemáticas o novedosas. Cuando decimos geografía aludimos a un tipo particular de conocimientos disciplinares - especulativos y profesionales que se forman mediante una experiencia única de aprendizaje, generalmente formal, que se evidencia en determinado tipo de percepciones, pensamientos, acciones y valoraciones; por ende, dada una experiencia de aprendizaje, solo es posible lograr o fallar en la adquisición de esta forma peculiar de pensar que forma parte (o no) del intelecto. Pero también alude a la disposición a manifestar cierta propensión valórica o actitudinal ligada a temáticas geográficas en las más diversas instancias de la vida. También pensar geográficamente puede ser concebido como acción (pensar) y producto (equivalente al cúmulo de conocimientos que también podríamos denominar teoría geográfica o pensamiento geográfico).

La formación académica y profesional requiere una base conceptual y teórica cuya pertinencia dependerá de la capacidad de quienes profesan esta ciencia de seguir produciendo pensamiento geográfico, (esto es, incrementando la masa de pensamiento geográfico como producto), por cuanto el logro de desempeños profesionales requiere de lineamientos teóricos que le otorguen sentido. Por otra parte, desde la teoría se reproducen metodologías, se diversifican los problemas de aplicación del conocimiento geográfico y se pueden construir las explicaciones que los resultados de las investigaciones iluminan.

Tim Ingold propuso re examinar la concepción tradicional que postula al conocimiento como el cimiento de la habilidad, entendida esta como un saber hacer, poniendo ambos factores en orden inverso al considerar que todo nuevo aprendizaje requiere la existencia de habilidades específicas precedentes (Ingold, 2012). Lo anterior equivale a decir que, si un estudiante cuenta con las condiciones externas y habilidades previas, (tales como capacidades lecto escritoras, dominio de vocabulario, comprensión lectora, conocimientos disciplinares, cartográficos, entre otros), aprenderá los conocimientos que se ponen a su disposición. Saber entonces equivale a poder, en tanto el sujeto dispone de mecanismos físicos o estructuras mentales que posibilitan la existencia de las capacidades requeridas para aprender y construir representaciones del mundo físico (Chomsky, 1983).

En términos de la profesionalidad, la aplicación de un tipo específico de conocimientos a la solución de problemas sociales relevantes requiere de una forma de pensar (nos referimos al pensamiento geográfico como acción). Se requiere avanzar en el logro de un tipo de pensar especialmente en las primeras etapas del proceso de formación académica y profesional. El como pensamos condiciona tanto la observación del entorno como la atención que ponemos en los objetos, sujetos, fenómenos y procesos; además, define la calidad de las preguntas que nos hagamos, las hipótesis que construyamos, los métodos que escojamos para responder las preguntas y las explicaciones que podamos elaborar (Harvey, 1983, 2006). 
Pensar geográficamente como recurso aporta a las posibilidades de que quien educa pueda presentar representando objetos, sujetos y fenómenos en una situación de aprendizaje. Tanto como acción como producto o resultado, este tipo de pensamientos posibilitan la simulación o el montaje, transfiriendo mediante el lenguaje (entre otros recursos puestos al servicio de los aprendizajes) los episodios, fragmentos o porciones de la realidad sobre la cual se pretende aplicar el análisis geográfico. Complementariamente, se hace necesario señalar que independientemente de los recursos puestos en juego, la enseñanza eficaz de cualquier cosa exige comprender que nuestras propias motivaciones, estrategias y capacidades para aprender no tienen porqué ser iguales a los alumnos que tenemos al frente, de hecho, no son las mismas. Aceptar lo anterior demanda que los académicos universitarios aceptemos que existe una gran variedad de opciones en cuanto a las razones, motivaciones y métodos para aprender, lo cual llevará indefectiblemente a estudiar por una parte, los procesos que intervienen en la construcción del conocimiento científico y en el aprendizaje de las ciencias y por otra, reflexionar acerca de qué concretamente significa pensar geográficamente, entendido como una cognición específica promovida por el dominio de conocimientos de carácter geográfico.

\section{Algunas conclusiones}

Pensar geográficamente tuvo en nuestro análisis varias lecturas. Primero, como acción que derivaba en la producción representacional; aquí pensar es equivalente a re - presentar lo que nos llega desde lo real. Dijimos que un pensamiento, entendido como un ente biológico - físico - químico - bioquímico - material producido en el cerebro deviene en la emergencia de una representación, que al ser parte de la conciencia, es como esta última, una propiedad emergente del funcionamiento del cerebro como un sistema no lineal.

Segundo, también dijimos que el pensar geográficamente generaba como resultado o producto un acervo que denominamos "pensamiento geográfico", entendido como un conjunto de estrategias o herramientas que nos permiten resolver tareas episódicas y a la vez como una especie de contenido proveniente de un proceso de aprendizaje generalmente formal de los recovecos de la ciencia geográfica, susceptible de ser aplicado en diversas instancias y etapas de la vida, con lo cual hicimos un guiño a la tesis de que el pensamiento geográfico funciona también en el largo plazo. Distinguimos entonces a la acción (pensar) del producto (pensamiento).

Tercero, nos situamos en el ámbito de los modelos. Aquí el pensar adquiere la condición de lugarizado y de replicación. Nos detuvimos en las condiciones o atributos que permiten que emerja otro pensamiento de uno precedente, que puede significar una recursión o profundización de la realidad captada desde el pensamiento original o bien una repetición, que como condición reconoce la posibilidad de que surja un pensamiento absolutamente diferente del inicial. La mente humana funciona modelando, por cuando requerimos para comprender ciertas homogeneidades o recursividades que nos permitan reconocer lo nuevo a partir de las propiedades de lo pre existente. En este sentido, pensar geográficamente es la continuidad evolutiva de la experiencia humana que busca explicar el mundo o entorno creativamente, modificando lo observado modificándose el observador. 
Sugerimos avanzar en la investigación acerca de los aspectos mentales comprometidos en la espacialidad, el impacto de la percepción y especialmente de la visión en la constitución de la realidad en los individuos, avanzar hacia la vinculación entre la Filosofía de la Mente, las neurociencias y la teoría geográfica. Todos estos son desafíos cuya resolución permitirá, entre otros fines posibles, la generación de ambientes más saludables, empáticos y humanizantes, o bien servir como insumo para que quienes enseñan geografía profundicen los conocimientos requeridos para tener la mejor idea posible de los factores influyentes en los aprendizajes de los sujetos con independencia de la edad, nivel de formación y temática.

\section{Bibliografía}

Aguilar, R. (2015). El sistema de posicionamiento cerebral: Premio Nobel en Fisiología y Medicina 2014 El pasado 6 de octubre de 2014, el comité Nobel del Instituto Karolino de Suec. Revista de La Facultad de Medicina (México).

Araya, S. U. (2002). Las representaciones sociales. Ejes teóricos para su discusión. Cuadernos de Ciencias Sociales 127. https://doi.org/10.1174/021347407782194425

Bale, J. (2006). Space, Place and Body Culture: Yi-Fu Tuan and a Geography of Sport. Geografiska Annaler. Series B, Human Geography. https://doi.org/10.2307/490831

Barry, A., \& Thrift, N. (2007). Gabriel tarde: Imitation invention and economy. Economy and Society. https://doi.org/10.1080/03085140701589497

Benjamin, W. (2007). Pequeña historia de la fotografía. In Obras II (pp. 377-402). Abada.

Benjamin, W. (2010). Sobre la facultad mimética. In Ensayos Escogidos (pp. 149-152). El cuenco de plata.

Bennett, Maxwell; Dennett, Daniel; Hacker, Peter; Searle, John, Robinson, D. N. (2003). Neuroscience and Philosophy: Brain, Mind, and Language. Blackwell Publishers.

Berman, M. (1987). El reencantamiento del mundo. Cuatro Vientos Editorial.

Bruner, J. (2003). Celebrando la divergencia: Piaget y Vigotsky. ITESO, Separata.

Casey, E. S. (1987). The place of space in the birth of the clinic. Journal of Medicine and Philosophy (United Kingdom). https://doi.org/10.1093/jmp/12.4.351

Chalmers, D. (2002). Philosophy of Mind: Classical and Contemporary Readings. Oxford University Press.

Chomsky, N. (1983). Reglas y representaciones. Fondo de Cultura Económica. 
Chopra, D. (2013). Reality and consciousness: A view from the East. Physics of Life Reviews. https://doi.org/10.1016/j.plrev.2013.11.001

Claxton, G. (1996). Structure, strategy and self in the fabrication of conscious experience. Journal of Consciousness Studies.

Claxton, G. (2002). Building Learning Power. In TLO Limited.

Claxton, G. (2007). Expanding young people's capacity to learn. British Journal of Educational Studies. https://doi.org/10.1111/j.1467-8527.2007.00369.x

Dennett, D. (2009). Intentional Systems Theory. In The Oxford Handbook of Philosophy of Mind. https://doi.org/10.1093/oxfordhb/9780199262618.003.0020

Dennett, D. C. (2012). Quining qualia. In Consciousness in Contemporary Science. https://doi. org/10.1093/acprof:oso/9780198522379.003.0003

Derrida, J. (1989). La escritura y la diferencia. Anthropos.

Didi-Huberman, G. (1997). Lo que vemos, lo que nos mira. In Bordes Manantial. https://doi.or$\mathrm{g} / 10.1029 / 91 \mathrm{JC} 03106$

Dretske, F. (1988). Explaining Behavior: Reasons in a World of Causes. MIT Press.

Edelman, G. (2006). Consciousness: The Remembered Present. Annals of the New York Academy of Sciences. https://doi.org/10.1111/j.1749-6632.2001.tb05711.x

Edelman, G. M. (2006). Second nature: Brain science and human knowledge. In Second Nature: Brain Science and Human Knowledge.

Escobar, A. (2003). Mundos y conocimientos de otro modo. Tabula Rasa. https://doi.org/10.3305/ nh.2012.27.4.5846

Foucault, M. (1978). Las palabras y las cosas. Una arqueología de las ciencias humanas. Siglo XXI.

Foucault, M. (1985). Saber y verdad. La Piqueta.

Foucault, M. (1992). Microfísica del poder. La Piqueta.

Foucault, M. (1993). 12. Verdad y poder. In Microfísica del Poder.

Foucault, M. (2000). Un Diálogo sobre el poder, y otras conversaciones. In El Libro de bolsillo.

Foucault, M. (2008). Seguridad, territorio, población. Cursos del College de France. Akal.

Grice, H. P. (1957). Meaning. Philosohical Review, 66, 377-388. 
Hall, S. (1980). Codificar y Decodificar. In Culture, Media, Language. Working Papers in Cultural Studies, 1972-79.

Harvey, D. (1983). Teoría, leyes y modelos en geografía. Alianza editorial.

Harvey, D. (2006). Editorial: The Geographies of Critical Geography. Transactions of the Institute of British Geographers, 31(4), 409-412.

Heidegger, M. (1994). Conferencias y Artículos. Ediciones del Serbal.

Ingold, T. (2012). Ambientes para la vida. Conversaciones sobre humanidad, conocimiento y antropología. Ediciones TRILCE.

Jackson, F. (2004). Representation and Experience. In Representation in Mind: New Approaches to Mental Representation. https://doi.org/10.4161/auto.7.3.14443

Jonas, H. (2000). El Principio Vida. Hacia una Biología Filosófica. Trotta.

Kanai, R., \& Tsuchiya, N. (2012). Qualia. In Current Biology. https://doi.org/10.1016/j.cub.2012.03.033

Latour, B. (1983). Give Me a Laboratory and I will Raise the World. In Science Observed: Perspectives on the Social Study of Science (pp. 141-170). SAGE Publications, Inc.

Latour, B. (2012). Gabriel tarde and the end of the social. In The Social in Question: New Bearings in History and the Social Sciences. https://doi.org/10.4324/9780203994535-15

Londoño, E. (2010). Desentrañando la lógica interna del Constructivismo Social de Vigotsky. (Pensamiento), (Palabra) y Obra, ISSN 2011-804X, Vol. 4, №. 4, 2010, Págs. 76-82.

Lussault, M. (2015). El hombre espacial. La construcción social del espacio humano. Amorrortu.

Maguire, E. A., Gadian, D. G., Johnsrude, I. S., Good, C. D., Ashburner, J., Frackowiak, R. S. J., \& Frith, C. D. (2000). Navigation-related structural change in the hippocampi of taxi drivers. Proceedings of the National Academy of Sciences of the United States of America. https://doi.org/10.1073/ pnas.070039597

Mainzer, K. (1994). Thinking in complexity. The Computacional Dynamics of Matter, MInds and Mankind. Springer.

Maturana, H. (1988). Ontology of observing: The biological foundations of self consciousness and the physical domain of existence. Cybernetics, American Society For Cybernetics Conference. https://doi.org/10.1017/СВ09781107415324.004

Maturana, H. (2002). Autopoiesis, Structural Coupling and Cognition : A history of these and other notions in the biology of cognition. Cybernetics \& Human Knowing. https://doi.org/10.1111/j.14711842.2008.00767.x 
Mauro, V. P., \& Edelman, G. M. (2002). The ribosome filter hypothesis. Proceedings of the National Academy of Sciences of the United States of America. https://doi.org/10.1073/pnas.192442499

Medina C., N. (2014). La ciencia cognitiva y el estudio de la mente. Revista de Investigación En Psicología. https://doi.org/10.15381/rinvp.v11i1.3890

Melero Martínez, J., \& Blanco Mayor, C. (1991). Walter Benjamin, el angelus novus como alegoría de la Historia. Ensayos: Revista de La Facultad de Educación de Albacete.

Mérenne-Schoumaker, B. (1994). Didactique de la géographie Organiser les apprentissages. In Perspectives didactiques.

Mérenne-Schoumaker, B. (2003). De la géographie des professeurs à la géographie de l'action: une place nouvelle dans l'enseignement secondaire. BELGEO.

Merlan, P., \& Jonas, H. (1967). The Phenomenon of Life: Toward a Philosophical Biology. Philosophy and Phenomenological Research. https://doi.org/10.2307/2105293

Moñivas Lázaro, A. (1994). Epistemología y representaciones sociales: concepto y teoría. Revista de Psicología General y Aplicada: Revista de La Federación Española de Asociaciones de Psicología.

Morin, E. (1999). La cabeza bien puesta. Repensar la reforma. Reformar el pensamiento. In Repensar la reforma. Reformar el pensamiento. Ediciones Nueva Visión.

Moscovici, S. (1984). The phenomenon of social representations. In Social Representations.

Nagel, T. (1974). What Is It Like to Be a Bat? The Philosophical Review. https://doi.org/10.2307/2183914

Nickerson, R.S, Perkins, D.N, Smith, E. E. (1994). Enseñar a pensar. Paidós.

Nida-Rümelin, M. (2009). Qualia: the Knowledge Argument. Stanford Encyclopedia of Philosophy.

Palm, G. (1981). Towards a theory of cell assemblies. Biological Cybernetics. https://doi.org/10.1007/ BF00342771

Panksepp, J. (1998). Affective neuroscience: the foundations of human and animal. In New York: Oxford University Press.

Panksepp, J. (2007). Affective Neuroscience and the Ancestral Sources of Human Feelings. In Consciousness and Cognition. https://doi.org/10.1016/B978-012373734-2/50014-5

Penrose, R. (1994). Las sombras de la mente. Hacia una comprensión científica de la consciencia. Crítica. 
Perner, J. (1997). Conocer la mente representacional. Paidós.

Philo, C. (2011). Michel Foucault. In Key Thinkers on Space and Place.

Pinker, S. (2012). El instinto del lenguaje: cómo crea el lenguaje la mente. In El libro universitario.

Porto-Gonçalves, C. (2009). De Saberes y de Territorios - diversidad y emancipación a partir de la experiencia latino-americana. Polis. Revista Latinoamericana.

Pozo, I. (1999). Aprendices y Maestros. La Nueva Cultura del Aprendizaje. Alianza editorial.

Rashid, A.J.; Yan, C.; Mercaldo, V.; Hsiang, H.; Park, S.; Cole, C.J.; De Cristofaro, A.; Yu, J.; Ramakrishnan, C.; Lee, SY., Deisseroth, K.; Frankland, P.W.; Josselyn, S. A., Rashid, A. J., Yan, C., Mercaldo, V., Hsiang, H. L., Park, S., Cole, C. J., Cristofaro, A. De, Yu, J., Ramakrishnan, C., Lee, S. Y., Deisseroth, K., Frankland, P. W., \& Josselyn, S. A. (2016). Competition between engrams influences fear memory formation and recall. Science. https://doi.org/10.1017/CBO9781107415324.004

Rizzolatti, G., \& Arbib, M. A. (1998). Language within our grasp. Trends in Neurosciences. https:// doi.org/10.1016/S0166-2236(98)01260-0

Rubenstein, A. (2009). Hans Jonas: A study in Biology and Ethics. Society. https://doi.org/10.1007/ s12115-008-9175-4

Ryle, G. (2005). El concepto de lo mental. Paidós.

Ryle, G. (2014). On thinking. In Essays and Reviews: 1959-2002.

Searle, J. R. (1985). Mentes, cerebros y ciencia. In Teorema. https://doi.org/10.1016/j.eujim.2012.02.002

Searle, J. R. (1994). Actos de habla :ensayo de filosofía del lenguaje. In Obras maestras del pensamiento contemporáneo.

Searle, J. R. (1996). El redescubrimiento de la mente. Crítica.

Searle, J. R. (2013). La Mente, una breve introducción. In Journal of Chemical Information and Modeling. https://doi.org/10.1017/СBO9781107415324.004

Shaw, G. L. (1986). Donald Hebb: The Organization of Behavior. In Brain Theory. https://doi. org/10.1007/978-3-642-70911-1_15

Shoemaker, S. (1991). Qualia and consciousness. Mind. https://doi.org/10.1093/mind/C.400.507

Singer, T., \& Lamm, C. (2009). The social neuroscience of empathy. Annals of the New York Academy of Sciences. https://doi.org/10.1111/j.1749-6632.2009.04418.x 
Stern, E. (2015). Embodied cognition: A grasp on human thinking. Nature. https://doi. org/10.1038/524158a

Tarde, G. (2013). Las leyes sociales. Gedisa Editorial.

Thagard, P. (2019). El cerebro. In La mente. https://doi.org/10.2307/j.ctvm7bd13.13

Thompson, E., \& Varela, F. J. (2001). Radical embodiment: Neural dynamics and consciousness. In Trends in Cognitive Sciences. https://doi.org/10.1016/S1364-6613(00)01750-2

Thrift, N. (2004). Thick time. In Organization. https://doi.org/10.1177/1350508404047255

Tirapu-Ustárroz, J., Pérez-Sayes, G., Erekatxo-Bilbao, M., \& Pelegrín-Valero, C. (2007). ¿Qué es la teoría de la mente? In Revista de Neurologia.

Toscano, A. (2007). Powers of pacification: State and empire in Gabriel Tarde. Economy and Society. https://doi.org/10.1080/03085140701589471

Tuan, Y.-F. (2007). Topofilia. Editorial Melusina S.L.

Varela, F. J. (1996). Neurophenomenology: A methodological remedy for the hard problem. Journal of Consciousness Studies.

Varela, F. J. (1999). Present-time consciousness. Journal of Consciousness Studies. 